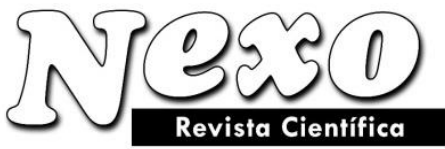

Vol. 34, No. 02, pp. 608-615/Junio 2021
ISSN-E 1995-9516

Universidad Nacional de Ingeniería COPYRIGHT @ (UNI). TODOS LOS DERECHOS RESERVADOS http://revistas.uni.edu.ni/index.php/Nexo https://doi.org/10.5377/nexo.v34i02.11546

\title{
Evaluación fisicoquímica y de propiedades funcionales del concentrado de proteínas de pescado de rio en función del $\mathrm{pH}$ del proceso
}

\section{Physicochemical and functional properties evaluation of the river fish protein concentrate gel as a function of the $\mathrm{pH}$ of the process}

\author{
José Raúl Medina *, Gustavo Alberto Pérez
}

Facultad de Ingeniería Química, Departamento de Proyecto y Sistemas de Gestión. Universidad Nacional del Litoral. Argentina

*irmedina@ fiq.unl.edu.ar

(recibido/received: 12-enero-2021; aceptado/accepted: 04-mayo-2021)

\section{RESUMEN}

El concentrado de proteínas de pescado CPP, es el músculo del pescado desmenuzado y exento de huesos, piel, carne oscura y de espinas, el que es lavado varias veces con agua y escurrido hasta la proporción de agua original, quedando lo que podría llamarse el material funcional o "surimi". El objetivo de este trabajo fue determinar cómo afectaba la variación del pH y la composición del agua de lavado a las muestras de CPP a partir del músculo de Sábalo (Prochilodus platensis). Los macrocomponentes estudiados fueron las proteínas totales, grasa total, humedad, cenizas totales, y la resistencia, el color y la capacidad de retención de agua (CRA) de los geles obtenidos a partir del CPP de sábalo. La resistencia del gel de CPP resultó para todos los tratamientos diferencias significativas a $\mathrm{p}<0.008$. La CRA para el tratamiento E4 y E6 no mostraron diferencias significativas. Y finalmente para el PCA se observa que el índice de blancura (B) para los tratamientos ensayados está en contraposición de los pH y de la CRA y a su vez está asociado a la Resistencia del gel de CPP en los primeros 2 componentes.

Palabras claves: surimi; sábalo; propiedades funcionales; PCA.

\begin{abstract}
The CPP fish protein concentrate is the muscle of the shredded fish, free of bones, skin, dark meat and bones, which is washed several times with water and drained to the original water proportion, leaving what could be called the functional material or "surimi". The objective of this work was to determine how the variation of the $\mathrm{pH}$ and the composition of the wash water affected the CPP samples from the muscle of "sabalo" (Prochilodus platensis). The macrocomponents studied were total proteins, total fat, moisture, total ash, and resistance, color and water retention capacity (CRA) of the gels obtained from the CPP of tarpon. The resistance of the CPP gel resulted in significant differences for all treatments at $\mathrm{p}<0.008$. The CRA for treatment E4 and E6 did not show significant differences. And finally, for the PCA it is observed that the whiteness index (B) for the tested treatments is in contrast to the $\mathrm{pH}$ and the CRA and in turn is associated with the resistance of the CPP gel in the first 2 components.
\end{abstract}

Keywords: surimi; sabalo; functional properties; PCA. 


\section{INTRODUCCIÓN}

El concentrado de proteínas de pescado CPP, es el músculo del pescado desmenuzado y exento de huesos, piel, carne oscura y de espinas, el que es lavado varias veces con agua y escurrido hasta la proporción de agua original, quedando lo que podría llamarse el material funcional (ó "surimi") del músculo de pescado (Suzuki, 1981).

El pescado es un alimento de captura estacional con un alto contenido en agua biológicamente activa, por lo que crudo se deteriora muy rápidamente, teniendo lugar alteraciones por crecimiento microbiano, actividad enzimática y por reacciones químicas originadas por interacciones entre sus nutrientes o con otros componentes. Estas reacciones se favorecen con la actividad del agua, la temperatura y el $\mathrm{pH}$, aparte de otras condiciones ambientales. De ahí que sea necesario proceder a su procesamiento para su conservación en las condiciones más idóneas y mantener sus propiedades sanitarias y nutritivas, de tal manera de prolongar su vida útil de consumo (Navarro, 1991b y 1991a).

El surimi es una alternativa a la situación planteada en el párrafo anterior. La palabra surimi es originaria de Japón y significa que es el músculo del pescado desmenuzado y exento de huesos, piel, carne oscura y de espinas, el que es lavado varias veces con agua y escurrido hasta la proporción de agua original, quedando lo que podría llamarse el material funcional (o producto intermedio) del músculo de pescado (Suzuki, 1981).

Esta base o intermediario es especial para la preparación de los productos análogos, de gran interés en el mundo occidental, y podría presentarse como alternativa industrial en Argentina. Sobre todo, para las especies ictícolas de agua dulce como el "sábalo" (Prochilodus platensis), de gran producción de biomasa, de muy bajo valor comercial para la venta directa, con el fin de proporcionar una forma distinta de presentación que permita una mejor conservación y por lo tanto extender su vida útil para su comercialización.

Los principales motivos del auge del desmenuzado de pescado (Surimi) a nivel mundial son: el mejor aprovechamiento del recurso ictícola desde el punto de vista nutricional, la posibilidad de estabilizar las proteínas durante el almacenamiento congelado de la pulpa mediante el agregado de crioprotectores, y por último la expansión de mercados existentes con nuevos productos atractivos que permitiría la ampliación del mercado de productos pesqueros (Manca y Trinchero, 1984).

Aunque la tecnología del surimi ha sido desarrollada y ampliamente investigada a partir de especies de mar sin valor comercial o sin explotación, actualmente se registran muy pocos trabajos que hayan utilizado como materia prima especies de pescado de agua dulce, y menos por supuesto, de alto contenido en grasa como es el sábalo, pero de una gran producción de biomasa.

Todos los avances realizados se han referido en general a pescado de mar y de bajo contenido en grasa. De todos modos, investigadores japoneses han obtenido notables mejoras en la tecnología del procesamiento de especies de alto contenido en grasa, logrando surimi con propiedades funcionales excelentes, lo que abre buenas expectativas para el aprovechamiento de tales especies (Nishioka y Tokunaga, 1990).

El objetivo de este trabajo fue determinar la aptitud del músculo del Sábalo (Prochilodus platensis), aprovechando experiencias previas de la tecnología surimi realizadas a partir del Surubí (Pseudoplatystoma coruscans) (Medina y Garrote, 2002a; 2002b), para producir surimi y determinar cómo afectaba la variación del pH y la composición del agua de lavado a las muestras de CPP a partir del músculo de Sábalo (Prochilodus platensis). Los macrocomponentes estudiados fueron las proteínas totales, grasa total, humedad, cenizas totales, y la resistencia, el color y la capacidad de retención de agua (CRA) de los geles obtenidos a partir del CPP de sábalo. 


\section{MATERIALES Y MÉTODOS}

\subsection{Planteo experimental}

Las experiencias realizadas se basaron en el trabajo de Medina y Garrote (2002a), en el cual se obtuvo la zona optima de las condiciones para obtener CPP de adecuada calidad funcional. Con éstas condiciones se llevó a cabo la obtención del CPP de Sábalo (Prochilodus platensis) a escala de laboratorio, sometiendo el desmenuzado de sábalo a tres tratamientos diferentes de lavado como lo expresa la TABLA 1 en función del pH y el componente de la solución. El tratamiento de Control como contraste se denominó como E2.

Tabla 1: Tratamientos de la etapa de lavado

\begin{tabular}{|c|c|c|c|c|}
\hline & \multicolumn{4}{|c|}{ Experimento } \\
\hline & E2 & E3 & E4 & E6 \\
\hline $1^{\circ}$ Ciclo & AGUA DESTILADA & $0.5 \%(\mathrm{p} / \mathrm{p}) \mathrm{NaHCO}_{3}$ & $0.05 \%(\mathrm{v} / \mathrm{v}) \mathrm{H}_{3} \mathrm{PO}_{4}$ & $0.05 \%(\mathrm{v} / \mathrm{v}) \mathrm{H}_{3} \mathrm{PO}_{4}$ \\
\hline $2^{\circ}$ Ciclo & AGUA DESTILADA & $0.5 \%(\mathrm{p} / \mathrm{p}) \mathrm{NaHCO}_{3}$ & $0.05 \%(\mathrm{v} / \mathrm{v}) \mathrm{H}_{3} \mathrm{PO}_{4}$ & $0.2 \%(\mathrm{p} / \mathrm{p}) \mathrm{NaHCO}_{3}$ \\
\hline $3^{\circ}$ Ciclo & $0.2 \% \mathrm{NaCl}(\mathrm{p} / \mathrm{p})$ & $0.2 \% \mathrm{NaCl}(\mathrm{p} / \mathrm{p})$ & $0.2 \% \mathrm{NaCl}(\mathrm{p} / \mathrm{p})$ & $0.2 \% \mathrm{NaCl}(\mathrm{p} / \mathrm{p})$ \\
\hline
\end{tabular}

2.2. Materiales, preparación del CPP de Sábalo congelado y sus geles

El músculo de pescado de río utilizado en estas experiencias fue el del sábalo (Prochilodus platensis). En Argentina, el Sábalo constituye una de las especies más comunes y de mayor biomasa en los cuerpos de aguas del río Paraná medio. Es una especie sub-explotada de bajo valor comercial por su carne, pero desarrolla una gran biomasa ya que por ejemplar tiene una capacidad reproductiva entre 300000 y 400000 huevos al año. Además, se registraron entre 1994 y 2004 exportaciones que pasaron de 2785 toneladas a 32000 toneladas de sábalo eviscerado (Espinach Ros y Sánchez, 2007). El Sábalo (Prochilodus platensis) fue obtenido de la región de pesca del río Paraná cercana a la ciudad de Santa Fe (Argentina).

El músculo blanco fue separado de las espinas, cuero, huesos y partes de músculo oscuro manualmente con cuchillo. El desmenuzado de sábalo fue preparado con una picadora eléctrica de carne con cuchilla y placa perforada para obtener un diámetro de partícula de $5 \mathrm{~mm}$. La metodología seguida para la preparación de las muestras y el sistema de lavado empleado fueron de acuerdo al trabajo realizado por Medina y Garrote, (2002a y 2002b).

Inmediatamente después del lavado y el prensado, el desmenuzado fue pesado y se le incorporó los crioprotectores sacarosa y sorbitol, en una relación 1:1 (p/p), de manera tal que alcance una concentración final del $8 \%$ en el peso final del bloque. Después que ésta masa fue mezclada manualmente con los crioprotectores se formaron bloques pequeños $(3 \mathrm{~cm}$. de diámetro y $12 \mathrm{~cm}$. de largo aproximadamente) y se congelaron por inmersión en Nitrógeno líquido por un tiempo calculado de 5 minutos, necesario para que el centro del bloque alcance la temperatura de $-21^{\circ} \mathrm{C}$, y luego almacenados a $-21^{\circ} \mathrm{C}$ en freezer hasta la determinación de los parámetros planteados en este trabajo, de acuerdo a la metodología recomendada por Lee (1986). Para la preparación de los 
geles de CPP también se procedió de la misma manera que el trabajo realizado por Medina y Garrote, (2002a)

\subsection{Métodos Analíticos}

Para caracterizar químicamente Proteínas totales se procedió por el método macro-Kjeldhal (AOAC, 1984) y humedad total (Lanier et. al. 1985), Lípidos totales fueron determinados por Folch et. al. (1957). Color: la medida de color fue hecha usando un Chroma Meter (Model CR310, Minolta Corp., Ramsey NJ, USA). Los valores del color fueron expresados según CIE: L*, $\mathrm{a}^{*} \mathrm{y} \mathrm{b}^{*}$. La efectividad del tratamiento para muestra blanca fue determinada por cálculo de luminosidad, replicando la medida por cuadriplicado, descripta como: Whiteness $(\mathrm{B})=100-$ $\left[\left(100-L^{*}\right)^{2}+a^{* 2}+b^{* 2}\right]^{1 / 2}$

Resistencia de los Geles: El análisis de textura de las muestras de CPP de sábalo fue realizado mediante una Máquina Universal Instron modelo 3344 siguiendo la metodología descripta por Medina et. al. (2002a).

Capacidad de retención de agua (CRA): muestras de geles cilíndricas fueron cortadas con un espesor de $5 \mathrm{~mm}$, pesadas y luego se siguió el método sugerido por Rawdkuen et. al. (2009).

\section{RESULTADOS}

En la Tabla 2 se muestran los resultados de las composiciones químicas promedios del músculo picado de Sábalo y de los CPP obtenidos bajo los tratamientos seleccionados.

En la Tabla 3 se puede observar los valores de $\mathrm{pH}$ medidos durante los tratamientos experimentados.

Tabla 2: Composición de las muestras de desmenuzado fresco y de CPP de

Sábalo obtenidos con distintos tratamientos

\begin{tabular}{cccccc}
\hline Macro-nutriente & $\begin{array}{c}\text { Desmenuzado } \\
\text { fresco } \\
\text { (gr. \%) }\end{array}$ & $\begin{array}{c}\text { E2 } \\
\text { (gr. \%) }\end{array}$ & $\begin{array}{c}\text { E3 } \\
\text { (gr. \%) }\end{array}$ & $\begin{array}{c}\text { E4 } \\
\text { (gr. \%) }\end{array}$ & $\begin{array}{c}\text { E6 } \\
\text { (gr. \%) }\end{array}$ \\
\hline Humedad & $72,58 \pm 2,54$ & $72,33 \pm 1,41$ & $74,49 \pm 0,88$ & $73,06 \pm 0,60$ & $73,26 \pm 0,59$ \\
Proteínas & $17,06 \pm 0,53$ & $14,61 \pm 0,39$ & $12,21 \pm 0,34$ & $13,50 \pm 1,09$ & $13,68 \pm 0,94$ \\
Grasa Total & $9,47 \pm 3,38$ & $5,27 \pm 0,69$ & $4,96 \pm 0,04$ & $4,82 \pm 0,74$ & $4,63 \pm 0,44$ \\
Cenizas Totales & $0,98 \pm 0,06$ & $0,40 \pm 0,01$ & $0,31 \pm 0,03$ & $0,52 \pm 0,09$ & $0,62 \pm 0,09$ \\
Hidratos de carbono & 0 & $8 \pm 0,01$ & $8 \pm 0,01$ & $8 \pm 0,01$ & $8 \pm 0,01$ \\
\hline
\end{tabular}

Valores promedios de 4 repeticiones con su desviación estándar 
Tabla 3: valores de $\mathrm{pH}$ medidos para cada experiencia

\begin{tabular}{cccc}
\hline EXP & $\mathrm{pH} 1^{\circ} \mathrm{Li}$ & $\mathrm{pH} 3^{\circ} \mathrm{LF}$ & $\mathrm{pH} \mathrm{MFL}$ \\
\hline E2 & $5,065 \pm 0,01$ & $6,85 \pm 0,1$ & $6,787 \pm 0,015$ \\
E3 & $8,503 \pm 0,294$ & $7,514 \pm 0,0125$ & $6,7625 \pm 0,015$ \\
E4 & $1,832 \pm 0,025$ & $5,323 \pm 0,145$ & $6,378 \pm 0,095$ \\
E6 & $2,175 \pm 0,03$ & $6,91 \pm 0,1$ & $6,855 \pm 0,09$
\end{tabular}

$p H$ de cuatro repeticiones con sus desv. Estándar. $p H 1^{\circ} \mathrm{Li}: \mathrm{pH}$ de la solución de lavado antes del $1^{\circ}$ lavado; $\mathrm{pH} 3^{\circ} \mathrm{LF}$ : pH de la solución al final del $3^{\circ}$ lavado y pH MFL: $p H$ del músculo lavado al final del $3^{\circ}$ lavado

En la Tabla 4 se pueden ver los resultados obtenidos del análisis de textura por medio de la evaluación de la resistencia de los geles de CPP para cada tratamiento realizado en este trabajo.

Tabla 4: Resultados del análisis de textura (valores promedios y su desviación estándar

\begin{tabular}{lrrrrrc}
\hline Exp. & $\begin{array}{l}\text { Resistencia } \\
\text { gel }(\mathrm{gr.cm})\end{array}$ & $\begin{array}{l}\text { Desv. } \\
\text { Est. }\end{array}$ & $\begin{array}{l}\text { Esfuerzo de } \\
\text { corte }(\mathrm{g})\end{array}$ & $\begin{array}{l}\text { Desv. } \\
\text { Est. }\end{array}$ & $\begin{array}{c}\text { Deformación } \\
(\mathrm{mm})\end{array}$ & $\begin{array}{l}\text { Desv. } \\
\text { est. }\end{array}$ \\
\hline E2 & 245,851 & 50,23 & 396,899 & 47,01 & 6,15 & 0,6 \\
E3 & 160,284 & 58,06 & 307,95 & 117,38 & 5,23 & 0,1 \\
E4 & 661,817 & 152,98 & 698,65 & 121,07 & 9,4 & 0,56 \\
E6 & 423,178 & 101,84 & 526,73 & 82,23 & 7,95 & 0,75 \\
\hline
\end{tabular}

Tabla 5 : Parámetros de color de los CPP, índices de blancura (B) y CRA

\begin{tabular}{lccccc}
\hline Exp. & $\mathrm{L}^{*}$ & $\mathrm{a}^{*}$ & $\mathrm{~b}^{*}$ & whiteness (B) & CRA \\
\hline E2 & $69,80 \pm 0,37$ & $1,66 \pm 0,24$ & $18,61 \pm 0,51$ & $64,48 \pm 0,32$ & $24,96 \pm 0,02$ \\
E3 & $73,36 \pm 0,79$ & $0,09 \pm 0,18$ & $15,65 \pm 0,96$ & $69,08 \pm 0,53$ & $19,60 \pm 0,02$ \\
E4 & $78,35 \pm 0,53$ & $1,91 \pm 0,38$ & $19,21 \pm 0,99$ & $70,97 \pm 0,48$ & $8,09 \pm 0,01$ \\
E6 & $74,44 \pm 0,95$ & $1,18 \pm 0,18$ & $17,63 \pm 0,71$ & $68,92 \pm 1,06$ & $9,21 \pm 0,004$ \\
\hline
\end{tabular}

En la Tabla 4, se puede observar los distintos valores que arrojó la medición de la resistencia de los geles según el tratamiento experimentado.

Los resultados se sometieron al análisis estadístico multivariante. En este sentido, se aprovecha el poder resolutivo de esta herramienta estadística no clásica, para la evaluación simultánea de todas las variables estudiadas, las que poseen tanto interés por sus propiedades físicas y químicas, como también presenta la nutricional.

La aplicación de las técnicas de análisis de componentes principales (PCA) y cuadrados mínimos parciales (PLS), permiten reducir la dimensión del sistema de datos (Esbensen, K y otros; 1998) La técnica de PCA utiliza un procedimiento matemático que transforma un conjunto de variables "respuesta" correlacionadas en un nuevo conjunto de variables no correlacionadas conocidas como "componentes principales"; éstas, determinan lo esencial de las variables originales, con una combinación lineal de ellas, indicando la dirección de máxima variancia de las muestras. 
Se sometió al análisis por PCA los datos obtenidos experimentalmente de las muestras, sobre las variables estudiadas. Dichos valores tipificaron respecto de la media.

Las correlaciones múltiples se exponen en la Tabla 6, y de ella surgen las significancias de mayor importancia entre todos los parámetros considerados. También se muestra en la figura 1 los resultados del PCA.

Tabla 6: Correlaciones múltiples

\begin{tabular}{|c|c|c|c|c|c|c|c|}
\hline & $\mathrm{pH} 3 \mathrm{LF}$ & $\mathrm{pH}$ MFL & $\begin{array}{c}\text { Proteínas } \\
\text { Totales }\end{array}$ & Humedad & Resistencia Gel & Whiteness (B) & CRA \\
\hline \multirow[t]{2}{*}{$\mathrm{pH} 3^{\circ} \mathrm{LF}$} & & 0,8567 & $-\mathbf{0 , 3 3 0 7}$ & 0,4070 & $-0,5674$ & $-0,4177$ & 0,2660 \\
\hline & & 0,0000 & 0,2109 & 0,1177 & 0,0219 & 0,1074 & 0,3193 \\
\hline \multirow[t]{2}{*}{$\mathrm{pH}$ MFL } & 0,8567 & & $-0,1023$ & 0,3122 & $-0,1700$ & $-0,3878$ & 0,6957 \\
\hline & 0,0000 & & 0,7061 & 0,2391 & 0,5290 & 0,1377 & 0,0028 \\
\hline \multirow[t]{2}{*}{ Prot. T } & $-\mathbf{0 , 3 3 0 7}$ & $-0,1023$ & & $-0,6591$ & 0,1598 & $-0,4532$ & 0,4324 \\
\hline & 0,2109 & 0,7061 & & 0,0055 & 0,5544 & 0,0779 & 0,0944 \\
\hline \multirow[t]{2}{*}{ Humedad } & 0,4070 & 0,3122 & $-0,6591$ & & $-0,1898$ & 0,2457 & $-0,1012$ \\
\hline & 0,1177 & 0,2391 & 0,0055 & & 0,4814 & 0,3591 & 0,7093 \\
\hline \multirow{2}{*}{$\begin{array}{c}\text { Resistencia } \\
\text { Gel }\end{array}$} & $-0,5674$ & $-\mathbf{0 , 1 7 0 0}$ & 0,1598 & $-0,1898$ & & $\mathbf{0 , 5 4 3 0}$ & 0,3354 \\
\hline & 0,0219 & 0,5290 & 0,5544 & 0,4814 & & 0,0297 & 0,2042 \\
\hline \multirow{2}{*}{$\begin{array}{l}\text { Whiteness } \\
\text { (B) }\end{array}$} & $-\mathbf{0 , 4 1 7 7}$ & $-0,3878$ & $-0,4532$ & 0,2457 & 0,5430 & & $-0,3079$ \\
\hline & 0,1074 & 0,1377 & 0,0779 & 0,3591 & 0,0297 & & 0,2460 \\
\hline \multirow[t]{2}{*}{ CRA } & 0,2660 & 0,6957 & 0,4324 & $-0,1012$ & 0,3354 & $-0,3079$ & \\
\hline & 0,3193 & 0,0028 & 0,0944 & 0,7093 & 0,2042 & 0,2460 & \\
\hline
\end{tabular}

Bigráfica

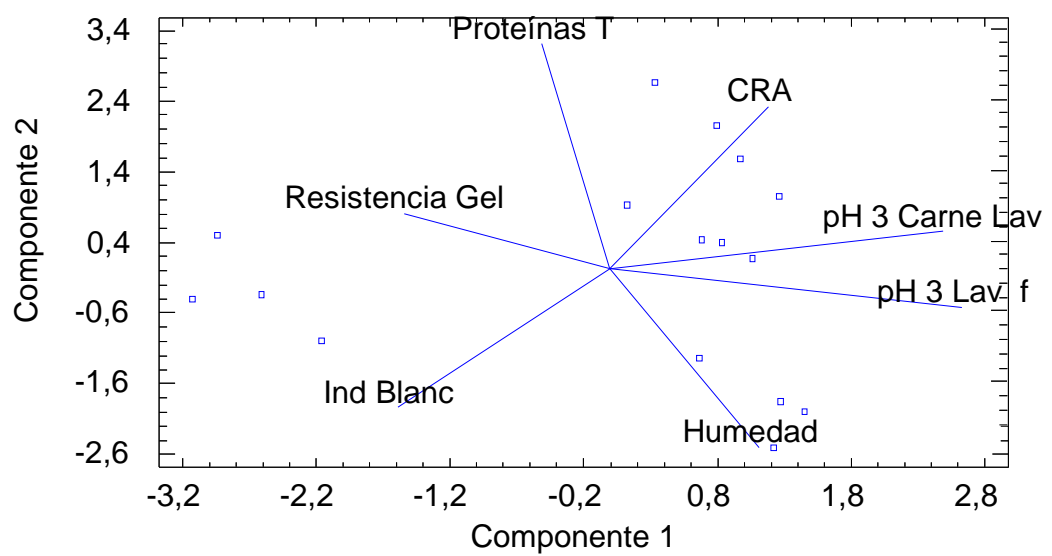

Figure 1 : PCA, Análisis de componentes principales 


\section{CONCLUSIONES}

Como conclusión de los resultados obtenidos es importante destacar las diferencias del sábalo analizado con los de otro músculo de pescado ya investigado por el grupo. Observándose que, si bien los porcentajes de proteínas son casi parecidos, existe una la relación entre el porcentaje de humedad y el de grasa total, dado que cuando está presente un mayor contenido de humedad en el músculo le corresponde una menor proporción de grasa total. Es importante resaltar que el músculo de sábalo demostró una adaptabilidad aceptable al proceso de obtención de surimi. Otras de las conclusiones es que el tenor graso en el surimi obtenido a partir de sábalo fue reducido desde un $9,5 \%$ en el filete fresco sin partes rojas u oscuras visibles a un promedio del $4 \%$ en el surimi aproximadamente. Este resultado es de suma importancia tecnológica en virtud del posterior almacenamiento congelado del surimi obtenido.

En cuanto a la diferencia de tratamientos, se puede observar que el agregado de $\mathrm{NaCl}$ al agua de lavado mejora el surimi obtenido en referencia a la menor retención de agua lográndose un surimi con un promedio del $73 \%$ de humedad final, lo cual es un objetivo tecnológico buscado y que beneficiará la etapa posterior que es la obtención de análogos de mariscos.

Con respecto a los experimentos en base a los resultados obtenidos se observan que todos los tratamientos dieron índices de blancura (B) con diferencias significativas a $\mathrm{p}<0.0001$. La resistencia del gel de CPP resulto que para todos los tratamientos presento diferencias significativas a $\mathrm{p}<0.008$. La CRA para el tratamiento E4 y E6 no mostraron diferencias significativas. Y finalmente para el PCA se observa que el índice de blancura (B) para los tratamientos ensayados esta en contraposición de los pH y de la CRA y a su vez este asociado a la Resistencia del gel de CPP en los primeros 2 componentes.

\section{AGRADECIMIENTO}

Los autores agradecen el apoyo financiero otorgado por la Universidad Nacional del Litoral de Santa Fe Argentina, para realizar el presente trabajo.

\section{REFERENCIAS}

A.O.A.C. International "Official Methods Of Analysis Of The Association Of Official Analytical Chemists (A.O.A.C.)", Ed. 165 ${ }^{\text {th }}, 1995$.

Esbensen, K., Schonkopf, S., Midtgaard, T., \& Guyot, D. (1998); Multivariate analysis in practice. CAMO ASA /Trondheim, Norway.

Espinach Ros, A. y Sánchez, R.P. (2007). Proyecto Evaluación del recurso Sábalo en el Paraná. En: SAGPyA Serie Pesca y Acuicultura: Informe de los resultados de la primera etapa 20052006 y medidas de manejo recomendadas. Secretaría de Agricultura, Ganadería, Pesca y Alimentos de la República Argentina. 
Folch, J., Lees, J.M. y Stanley, G.H.S. (1957). A simple method for the isolation and purification of total lipids from animal tissues. Journal of Biological Chemistry 226(1), 497-509.

Lanier, T.C., Hamann, D.D., y Wu, M.C. (1985). Development of methods for quality and functionality assessment of surimi and minced fish to be used in gel type food products. En: Final Report for Alaska Fisheries Development Foundation, Inc.; Anchorage, Alaska.

Manca, E. y Trinchero, J. (1984). Desmenuzado de pescado: tecnología y posibles usos. Parte I. La Industria Cárnica Latinoamericana 56, 41-50.

Medina, J.R. y Garrote, R.L. (2002a). Determining washing conditions during the preparation of frozen surimi from Surubí (Pseudoplatystome coruscans) using response surface methodology. Journal of Food Science 67(3), 1455-1461.

Medina, J.R. y Garrote, R.L. (2002b). Effect of two cryoprotectant mixtures on frozen surubí surimi. Brazilian Journal of Chemical Engineering 19(4), 419-424.

Navarro, M.P. (1991a). Valor nutritivo del pescado. I- Pescado fresco. Revista de Agroquímica y Tecnología de Alimentos 31(1), 330-342.

Navarro, M.P. (1991b). Valor nutritivo del pescado. II - Pescado elaborado. Revista de Agroquímica y Tecnología de Alimentos 31(4), 459-472.

Nishioka, F. y Tokunaga, T. (1990). Development of new leaching technology and a system to manufacture high quality surimi. Proceedings of the international Institute of Refrigeration 3, 123-130.

Rawdkuen, S. Samart Sai-Ut, Saisunee Khamsorn, Manat Chaijan, Soottawat Benjakul (2009). "Biochemical and gelling properties of tilapia surimi and protein recovered using an acid-alkaline process". Food Chemistry 112, p. 112-119

Susuki, T. (1981). Fish and krill protein: processing technology. Applied Science Publishers Ltd., England. 